\title{
Scanning second-harmonic/third-harmonic generation microscopy of gallium nitride
}

\author{
Chi-Kuang Sun, ${ }^{\text {a) }}$ Shih-Wei Chu, and Shi-Peng Tai \\ Department of Electrical Engineering and Graduate Institute of Electro-Optical Engineering, \\ National Taiwan University, Taipei, Taiwan, 10617 Republic of China \\ Stacia Keller, Umesh K. Mishra, and Steven P. DenBaars \\ Department of Electrical and Computer Engineering, University of California, Santa Barbara, \\ California 93106
}

(Received 22 May 2000; accepted for publication 16 August 2000)

\begin{abstract}
Scanning second-harmonic generation and third-harmonic generation microscopy of a gallium nitride $(\mathrm{GaN})$ sample was demonstrated using a femtosecond Cr:forsterite laser. Taking advantage of the electric-field enhanced second-harmonic generation effect and bandtail state resonance effect, the obtained second-harmonic and third-harmonic generation microscopic images revealed the piezoelectric field and bandtail state distributions in a GaN sample. (C) 2000 American Institute of Physics. [S0003-6951(00)03141-7]
\end{abstract}

The recent demonstration of high-brightness lightemitting diodes and laser diodes has established the III-V nitrides as key materials for optoelectronics operating in the green-ultraviolet wavelength range. ${ }^{1}$ Due to the lack of a suitable lattice-matched substrate, the optical behaviors of the grown GaN layer were found to be strongly affected by high densities of defect states and large residue piezoelectric fields. ${ }^{1,2}$ Defect-related strong yellow luminescence and high density of bandtail states are frequently observed in most $\mathrm{GaN}$ materials. The optical behaviors of InGaN quantum wells grown on top of $\mathrm{GaN}$ are also modified by the existence of a strong piezoelectric field and high defect densities. A considerable amount of studies have been devoted to clarify the roles of defect versus piezoelectric field in the photoluminescence (PL) spectra that is related to the laser mechanism. In this letter, we present a microscopic technique that can be used to map the electric field distributions as well as the defect-related bandtail state distributions with scanning second-harmonic-generation (SHG) and thirdharmonic-generation (THG) microscopies.

Our study was motivated by the recent report on the observation of strong electric-field induced second-harmonic (EFISH) generation in bulk GaN. ${ }^{3}$ Miragliotta et al. have experimentally determined the second order susceptibility $\chi^{(2)}$ in bulk GaN and have found increased nonlinearities when a large direct current (dc) electric field was applied to the surface of a GaN film. ${ }^{3}$ With a wurzite structure and a 6 $\mathrm{mm}$ group symmetry, $\mathrm{GaN}$ is an uniaxial crystal with the effective $\chi^{(2)}$ expressed as ${ }^{3}$

$\chi_{i j k}^{(2)^{\mathrm{eff}}}(2 \omega: \omega, \omega)=\chi_{i j k}^{(2)}(2 \omega: \omega, \omega)+3 \chi_{i j k l}^{(3)}(2 \omega: \omega, \omega, 0) E_{l}$,

where the second term in Eq. (1) represents the EFISH effect with $E_{l}$ as the strength of the dc electric field. Miragliotta et al. have reported a value of $1.3 \times 10^{-11} \mathrm{~m} / \mathrm{V}$ for $\chi_{z x x}^{(2)}(2 \omega: \omega, \omega)$ and a value of $5.3 \times 10^{-19} \mathrm{~m}^{2} / \mathrm{V}^{2}$ for

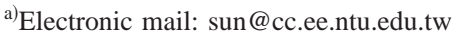

$\chi_{z x x z}^{(3)}(2 \omega: \omega, \omega, 0)$ at a wavelength around the midgap of GaN. According to their study, a positive electric field on the order of $10 \mathrm{kV} / \mathrm{cm}$ can increase $10 \%$ value of the effective $\chi^{(2)}$ and $20 \%$ value of the generated SHG power. This strong EFISH effect can be attributed to the large $\chi^{(3)}$ process in $\mathrm{GaN}$. Recent studies of $\chi^{(3)}$ process in $\mathrm{GaN}$ indicated strong third-order nonlinearities in $\mathrm{GaN}$ materials, including nonlinear refractive index, ${ }^{4}$ two photon absorption, ${ }^{5}$ and thirdharmonic generations. ${ }^{6}$ Miragliotta et al. have experimentally determined the third order susceptibility $\chi_{x x x x}^{(3)}(3 \omega: \omega, \omega, \omega)$ in bulk GaN to be on the order of 3.8 $\times 10^{-19} \mathrm{~m}^{2} / \mathrm{V}^{2}$ at a wavelength one third of the band gap energy. ${ }^{6}$ Piezoelectric field enhanced second-order nonlinear optical susceptibility was also observed in InGaN/GaN quantum wells. ${ }^{7,8}$ EFISH was previously used to probe the voltage across a biological membrane $\mathrm{e}^{9}$ and was also observed in polysilanes. ${ }^{10}$ By scanning the SHG power after the GaN sample, we are thus able to map the residue piezoelectric field distribution in a $\mathrm{GaN}$ layer.

Even though it seems straight forward to use a femtosecond Ti:sapphire laser to perform this mapping, the close resonance wavelength will make the SHG power sensitive to the defect related bandtail states. In this case, the scanning SHG image will not be able to separate the effects of piezoelectric field from defect distribution. In our study, we performed the scanning SHG microscopy using a femtosecond $\mathrm{Cr}$ :forsterite laser with a wavelength of $1230 \mathrm{~nm}$, which is off-resonant from the GaN band gap. By using a wavelength around $1200 \mathrm{~nm}$ we cannot only use SHG intensity to map the piezoelectric field distribution, but can also obtain close resonant THG signals, which would be enhanced by the bandtail states and can be used to probe their distribution.

Figure 1 shows the experimental setup. The experiments were performed using a femtosecond Cr:forsterite laser, which was pumped by a $7 \mathrm{~W} \mathrm{Nd:YVO}$ laser with an output wavelength centered at $1230 \mathrm{~nm}$ with a pulsewidth of $150 \mathrm{fs}$. The maximum average output power was around $350 \mathrm{~mW}$ with $125 \mathrm{MHz}$ repetition rate. The output infrared (IR) laser beam was first shaped by a telescope and then focused into a 


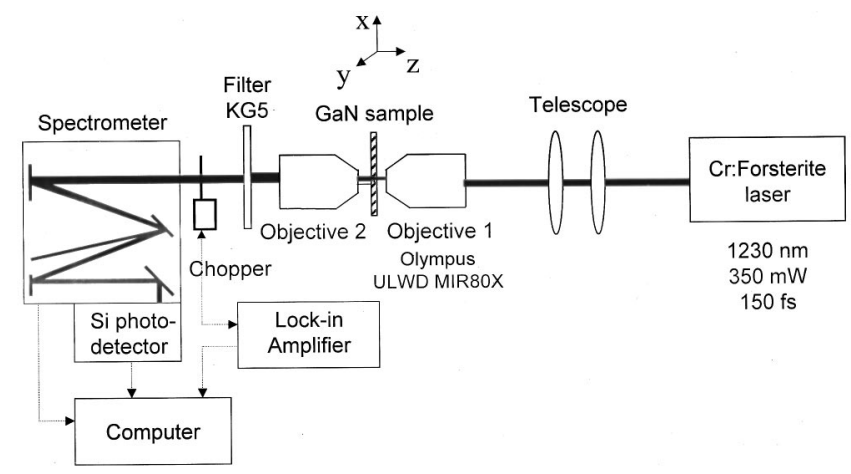

FIG. 1. Experimental layout for the scanning SHG/THG microscopy.

GaN sample with an $80 \times$ IR applicable objective. The focused spot size was around $1.3 \mu \mathrm{m}$. Visible SHG with red color and THG with purple color were collected and collimated using an opposing objective. Due to the nature of harmonic generations, no absorption is needed in the generation process, which can avoid on focus damages and bleaching compared with multiphoton PL microscopy. We filtered out the fundamental IR beam with an optical glass filter. The collected red/purple light was then directed into a spectrometer for the spectrum measurements. Due to the relatively large harmonic generation signals, an unamplified Si photodetector will be suitable for the light detection. Figure 2 shows some typically measured SHG/THG spectra. The laser spectrum is also provided for comparison. With a fundamental wavelength centered around $1230 \mathrm{~nm}$, symmetric SHG/ THG spectra centered on 615 and $410 \mathrm{~nm}$ can be observed.

The scanning SHG/THG images can then be obtained by moving the GaN sample in the plane ( $x-y$ plane) perpendicular to the sample $c$ axis (extraordinary axis, or $z$ axis) by using a stepping motor-driven stage. Three-dimensional images are obtainable through $x-y-z$ scans. SHG image technique was introduced by Gannaway and Sheppard ${ }^{11}$ and was applied to imaging inorganic nonlinear materials, ${ }^{11}$ molecular thin films, ${ }^{12}$ and animal tissues. ${ }^{13}$ THG imaging technique was proposed recently and was demonstrated in fiber ${ }^{14}$ and biological specimen. ${ }^{15}$ In this letter we extend these techniques to the studies of semiconductors. For the application in GaN imaging, there are several problems we have to consider. Due to the fact that most GaN samples are grown in the direction of $c$ axis, a scanning in the plane perpendicular to the sample $c$ axis would be preferred. However there are only seven $\chi^{(2)}$ components existing in wurzite structures $(x z x=y z y, x x z=y y z, z x x=z y y$, and $z z z)$. In order to generate SHG in GaN, an angle between beam propagation direction and the $c(z)$ axis is required. To map out the EFISH signal induced by a piezoelectric field in $z$ direction, similar requirement will be hold. This SHG requirement obviously violates the preferred scanning arrangement that requires a $0^{\circ}$ angle between the beam propagation direction and the sample $c$ axis. The problem was solved by using a tightly focused laser beam with the $80 \times$ objective, which would induce curved wave fronts around the focal point and provided the required optical-field $z$ components. For THG microscopy, the $\chi_{x x x x}^{(3)}$ component will provide the necessary THG process for a laser beam with $x$-polarized light. How-
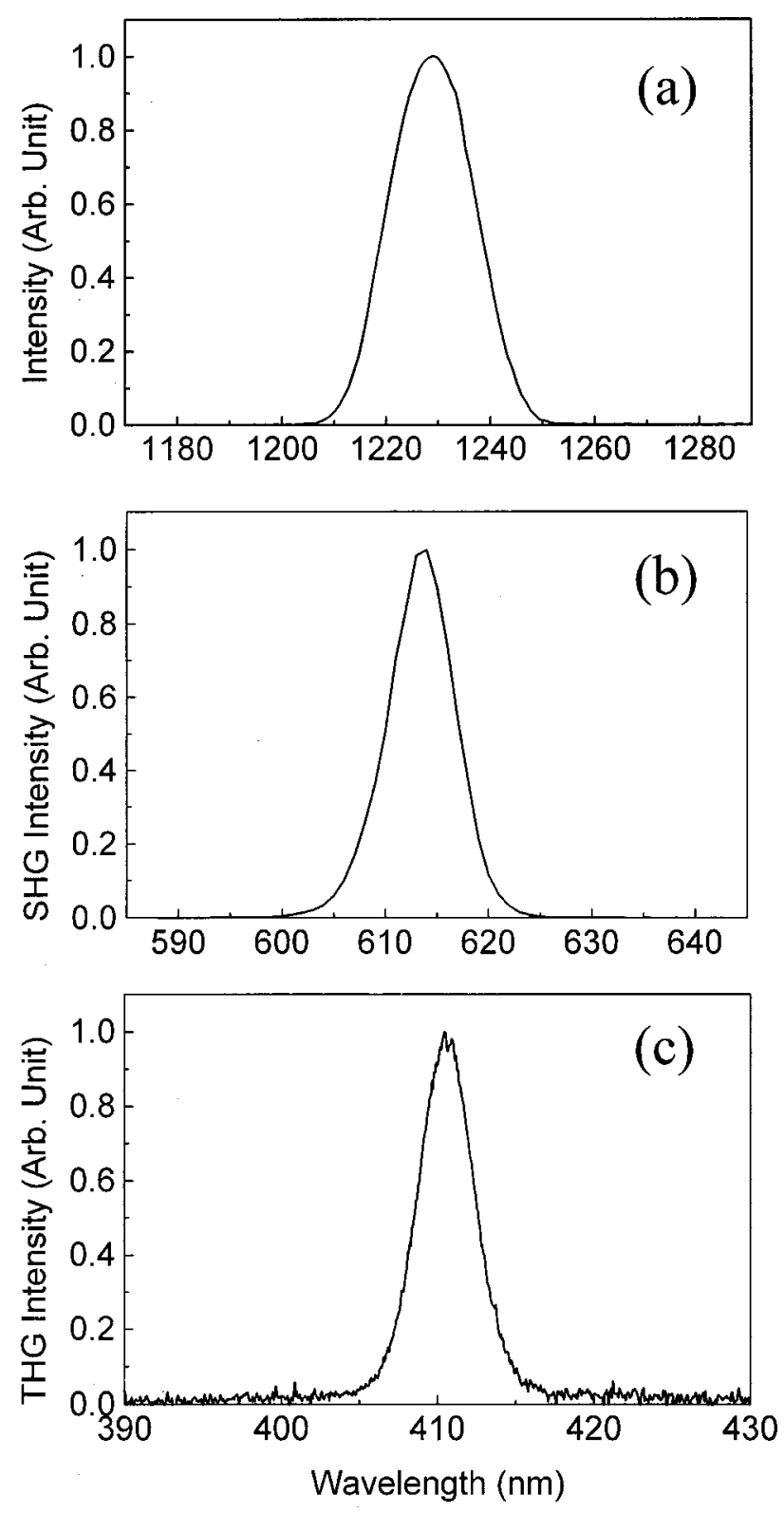

FIG. 2. Spectra for (a) IR laser beam, (b) SHG, and (c) THG signals from a 2.5- $\mu$ m-thick bulk GaN.

ever, the positive dispersion in GaN will limit the effective THG generation only in the surface layer thinner than the confocal parameter. The large refractive index difference ${ }^{16}$ between the fundamental and the THG light would also limit the coherent length of the THG process to be on the order of only $1 \mu \mathrm{m}$. The latter process will reduce the THG signals from a thicker sample. This situation was improved in our experiments due to the close resonant excitation scheme. Figure 3 shows typically measured SHG and THG average power versus input IR average power for a $2.5-\mu \mathrm{m}$-thick bulk GaN Hall-measurement sample ${ }^{5}$ by using the $80 \times$ objective with laser beam propagating in the $c$ axis $(0$ incident angle). With a $45 \mathrm{~mW}$ input power, a SHG output power of $140 \mathrm{nW}$ and a THG output power of $10 \mathrm{nW}$ can be obtained. Open circles are the experimental data and the dashed lines correspond to square/cubic dependence of the output-input relations. Slopes of 2 and 3 can be found in the $\log -\log$ plots confirming the SHG and THG origins of the measured signals. A value of $3 \times 10^{-12} \mathrm{~m} / \mathrm{V}$ for $\chi_{\text {eff }}^{(2)}(2 \omega: \omega, \omega)$ and a 

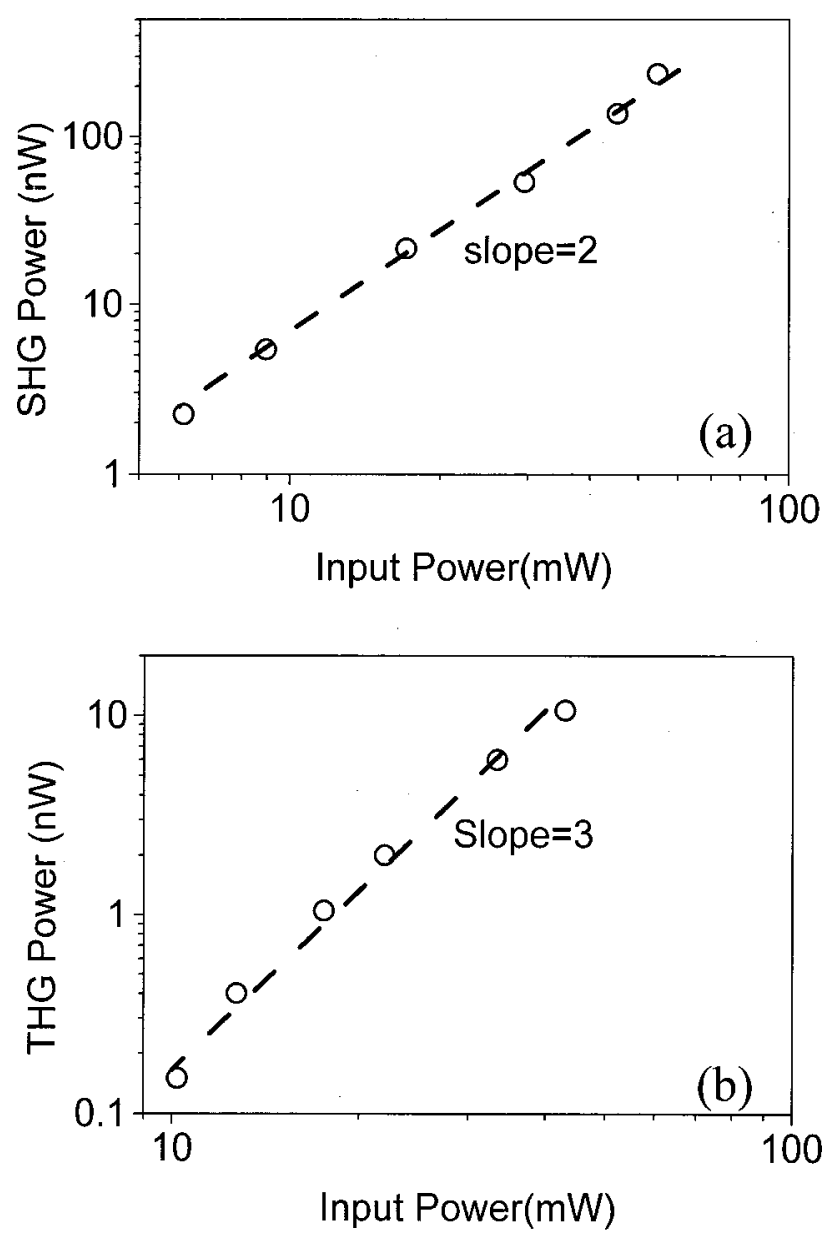

FIG. 3. (a) Average SHG power vs average input IR power and (b) average THG power vs average input IR power in a $2.5-\mu \mathrm{m}$-thick GaN sample.

value of $1 \times 10^{-20} \mathrm{~m}^{2} / \mathrm{V}^{2}$ for $\chi_{x x x x}^{(3)}(3 \omega: \omega, \omega, \omega)$ can thus be derived for our specific experimental wavelengths.

Figure 4 shows the scanning SHG microscopic image and its corresponding scanning THG microscopic image of the $2.5 \mu \mathrm{m}$-thick GaN Hall-measurement sample (paradermal view). A transmission image taken with the fundamental IR beam is also presented for comparison. The image was taken from the edge of the Hall-measurement sample with an oval defect. The GaN sample shows excellent lateral uniformity in the SHG except on the area around the oval defect, where decreased SHG intensity can be observed. It is interesting to notice that the heart-shaped area generating weak SHG signals around the oval defect emitted strong THG signals. Our data suggests that in this area the THG signals were resonantly enhanced by the increased bandtail states around the oval defects. At the same time these defects reduced the residue piezoelectric field by strain relaxation or by a space charge screening effect, resulting in the decreased SHG signals in the same area. It is also interesting to compare the scanning SHG/THG microscopy with other scanning multiphoton microscopy. We have previously performed twophoton PL microscopy in the same Hall-measurement sample. ${ }^{5}$ Our previous study showed that the heart-shaped
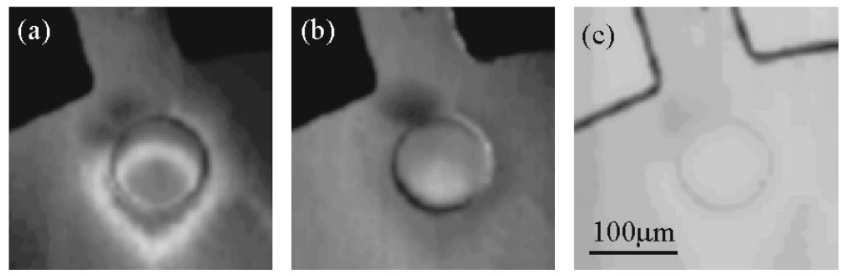

FIG. 4. (a) Scanning THG, (b) SHG, and (c) corresponding transmission images of the $2.5-\mu \mathrm{m}$-thick GaN Hall-measurement sample.

area generating strong THG signals emitted strong yellow luminescence with suppressed band edge PL. ${ }^{5}$ Our microscopy studies thus indicate the connections between suppressed band edge PL, increased yellow luminescence, increased bandtail states, and decreased piezoelectric field. We also observed a dark region above the oval defect in both the SHG and THG images. This area should correspond to the region with degraded crystal quality.

In summary, scanning SHG and THG microscopy of $\mathrm{GaN}$ was demonstrated. Taking advantages of the electricfield enhanced SHG effect and the off-resonant SHG processes, the obtained scanning SHG image reveals the distribution of residue piezoelectric field. Taking advantage of the bandtail-state resonance effect, the obtained THG microscopic image, on the other hand, reveals bandtail state distributions. These SHG/THG microscopies not only exhibit superior images of the piezoelectric field as well as the defectrelated bandtail state distributions in $\mathrm{GaN}$, combining with multiphoton PL microscopy these techniques can also provide interesting details that are not readily available before.

This project is sponsored by National Science Council of Taiwan, Republic of China under Grant No. NSC 89-2112M-002-044.

${ }^{1}$ S. Nakamura and G. Fasol, The Blue Laser Diode (Spriger, Berlin, 1997).

${ }^{2}$ B. J. Skromme, H. Zhao, D. Wang, H. S. Kong, M. T. Leonard, G. E. Bulman, and B. J. Molnar, Appl. Phys. Lett. 71, 829 (1997).

${ }^{3}$ J. Miragliotta and D. K. Wickenden, Phys. Rev. B 53, 1388 (1996).

${ }^{4}$ Y. L. Huang, C.-K. Sun, J.-C. Liang, S. Keller, M. P. Mack, U. K. Mishra, and S. P. DenBaars, Appl. Phys. Lett. 75, 3524 (1999).

${ }^{5}$ C.-K. Sun, J.-C. Liang, J.-C. Wang, F.-J. Kao, S. Keller, M. P. Mack, U. Mishra, and S. P. DenBaars, Appl. Phys. Lett. 76, 439 (2000).

${ }^{6}$ J. Miragliotta and D. K. Wickenden, Phys. Rev. B 50, 14960 (1994).

${ }^{7}$ H. Schmidt, A. C. Abare, J. E. Bowers, S. P. DenBaars, and A. Imamoglu, Appl. Phys. Lett. 75, 3611 (1999).

${ }^{8}$ A. Liu, S.-L. Chuang, and C. Z. Ning, Appl. Phys. Lett. 76, 333 (2000).

${ }^{9}$ O. Bouevitch, A. Lewis, I. Pinevsky, J. P. Wuskell, and L. M. Loew, Biophys. J. 65, 672 (1993).

${ }^{10}$ H. Kishida, T. Hasegawa, Y. Iwasa, T. Koda, Y. Tokura, H. Tachibana, M. Matsumoto, S. Wada, T. T. Lay, and H. Tashiro, Phys. Rev. B 50, 7786 (1994).

${ }^{11}$ J. N. Gannaway and C. J. R. Sheppard, Opt. Quantum Electron. 10, 435 (1978).

${ }^{12}$ T. Jentsh, H. J. Jupner, S. H. Ashworth, and T. Elsaesser, Opt. Lett. 21, 492 (1996).

${ }^{13}$ I. Freund, M. Deutsch, and A. Sprecher, Biophys. J. 50, 693 (1986).

${ }^{14}$ Y. Barad, H. Eisenberg, M. Horowitz, and Y. Silberberg, Appl. Phys. Lett. 70, 922 (1997).

${ }^{15}$ M. Müller, J. Squier, K. R. Wilson, and G. J. Brakenhoff, J. Microsc. 191, 266 (1998).

${ }^{16}$ H. Y. Zhang, X. H. He, Y. H. Shih, M. Schurman, Z. C. Feng, and R. A. Stall, Appl. Phys. Lett. 69, 2953 (1996). 